\title{
Wide Intraoral Surgical Access to the Inferior Alveolar Nerve During Cryotherapy at the Infratemporal Fossa: Technical Modification
}

\author{
Constantinus Politis, MD, DDS, MHA, MM, PhD, ${ }^{*}$ Maria Piagkou, DDS, MD, MSc, PhD,$\dagger$ \\ Ivo Lambrichts, DDS, PhD, $\ddagger$ and Jimob Olubanwo Agbaje, BDS, DMD, MMI, PhD
}

\begin{abstract}
Trigeminal neuralgia is characterized by unilateral pain in the region supplied by the sensory distribution of the fifth cranial nerve. Pharmacologic therapy is an adequate initial treatment option in $75 \%$ of patients. When the Jannetta surgical operation is not available or not indicated and when conservative treatment fails to relieve the pain or the medication has to be discontinued because of side effects, one of the remaining surgical options is cryosurgery in the peripherally distributed nerves that emanate from the trigeminal nerve. This technical note describes a perioperative method for exposing and mobilizing the inferior alveolar nerve (IAN) from its bony canal. This approach provided easy access to infratemporal fossa structures during cryotherapy. This technique represented a further development of the technique previously described by the authors. This method ensured direct visualization of the IAN and wide access to theinfratemporal fossa during IAN cryotherapy.
\end{abstract}

(C) 2018 American Association of Oral and Maxillofacial Surgeons

J Oral Maxillofac Surg 76:2090.e1-2090.e5, 2018

Trigeminal neuralgia is a facial pain syndrome with an estimated prevalence of 0.03 to $0.30 \% .{ }^{1,2}$ It is characterized by unilateral pain (which can become recurrent and chronic) in the region supplied by the sensory distribution of the fifth cranial nerve. Pharmacologic therapy is an adequate initial treatment option in $75 \%$ of patients. ${ }^{3,4}$ The preferred surgical approach is the Jannetta operation when there is an established neurovascular conflict at the root entry zone of the nerve. ${ }^{5,6}$ When this surgical option is not available or not indicated and when conservative treatment fails to relieve the pain or the medication must be discontinued because of side effects, one of the remaining surgical options is cryosurgery in the peripheral nerve distribution that emanates from the trigeminal nerve. In 1976, Lloyd et $\mathrm{al}^{7}$ first described cryosurgery as a treatment for trigeminal neuralgia to eliminate pain in peripheral nerves. Good results were achieved with an open nitrogen spray directed at the infraorbital nerve. However, that approach requires surgical exposure of the nerve, and when inaccurately applied, the spray is likely to damage adjacent tissue. Moreover, even with the newly developed cryoprobe, wider access
*Professor and Head, Oral and Maxillofacial Surgery Department, OMFS-IMPATH Research Group; Department of Imaging and Pathology, Faculty of Medicine, Katholieke Universiteit Leuven; Department of Oral and Maxillofacial Surgery, University Hospitals Leuven, Leuven, Belgium.

$\nmid$ Professor and Head, Division of Anatomy, National and Kapodistrian University of Athens, Athens, Greece.

$\ddagger$ Professor and Head, Laboratory of Morphology Research Group, Faculty of Medicine, Hasselt University; Biomedical Research Institute, Laboratory of Morphology, Hasselt University, Campus Diepenbeek, Diepenbeek, Belgium.

$\S$ Postdoctoral Research Fellow, OMFS-IMPATH Research Group, Department of Imaging and Pathology, Faculty of Medicine,

\footnotetext{
Katholieke Universiteit Leuven; Department of Oral and Maxillofacial Surgery, University Hospitals Leuven, Leuven, Belgium. Conflict of Interest Disclosures: None of the authors have a relevant financial relationship(s) with a commercial interest.

Address correspondence and reprint requests to Dr Agbaje: OMFS-IMPATH Research Group, Department of Oral and Maxillofacial Surgery, University Hospitals of Leuven, Kapucijnenvoer 33, 3000 Leuven, Belgium; e-mail: joagbaje@gmail.com Received January 172018 Accepted May 302018 (C) 2018 American Association of Oral and Maxillofacial Surgeons 0278-2391/18/30514-7 https://doi.org/10.1016/i.joms.2018.05.039
} 

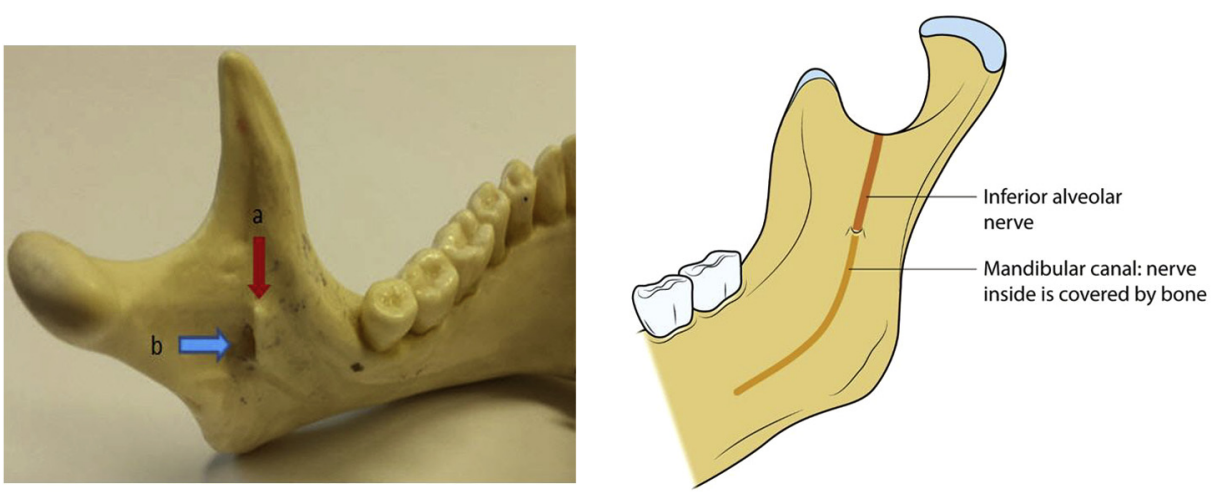

FIGURE 1. Positions of the lingula of the mandible, mandibular foramen, and inferior alveolar nerve. Left, Lateral view of the mandible shows the locations of $(a)$ the lingula of the mandible (red arrow) and (b) the mandibular foramen (blue arrow). Right, Diagram of a lateral view of the mandible shows the path of the inferior alveolar nerve and its point of entry into the mandibular canal.

Politis et al. Surgical Access to IAN During Cryotherapy. J Oral Maxillofac Surg 2018.

is required, particularly in patients who require a redo cryotherapy below a fibrotic region of the nerve resulting from a previous surgery or cryotherapy at the infratemporal fossa. Direct visualization allows a clear field of view of the operation site, facilitates proper application of the cryoprobe, and ensures the inferior alveolar nerve (IAN) can be distinguished from the lingual nerve. . $^{8-10}$

This technical note describes a perioperative method for exposing and mobilizing the IAN from its bony canal. This approach provided easy access to structures in the infratemporal fossa region during cryotherapy. This technique represented a further development of the technique previously described by the authors for direct visualization of the IAN during instrumentation and surgical procedures at the mandibular foramen. ${ }^{11}$ This modified method ensured direct visualization of the IAN and wide access at the infratemporal fossa during IAN cryotherapy.

\section{Surgical Technique}

The IAN is prone to injury at its point of entry into the mandibular foramen during surgical operations that involve the infratemporal fossa with an intraoral approach. It is necessary to locate the lingula, which lies above the opening of the mandibular canal, and ascertain where the IAN enters the canal (Fig 1). Often, the lingula is not directly visible because of the inclination of the ascending ramus or a thick anterior ridge at the ascending ramus. In those cases, the lingula can be palpated behind the ramus with a nerve hook, but not directly viewed. Also, the extent to which the nerve can be mobilized at the ascending ramus depends on the vertical and sagittal location of the mandibular foramen. The higher and more posterior the foramen, the less the nerve can be mobilized. Fibrosis and scar formations from previous surgeries also can limit access to the IAN. Therefore, the authors developed a technique to address these challenges.

Similar to the technique used in the classic sagittal split osteotomy, ${ }^{12}$ access to the lingula was achieved by first identifying the lingula with a nerve hook (Fig 2).

Clamp and Dumbach retractors were positioned at the coronoid process to protect the nerve. A suction device was positioned parallel and approximately $1 \mathrm{~cm}$ cranial to the Dumbach retractor. Anterior to the IAN, the entire cortical bone of the inner part of the ascending ramus was removed with a thick round drill while the Dumbach retractor protected the nerve (Fig 3). The amount of bone removed was approximately the width of the Dumbach retractor.

With the Dumbach retractor in place and a proper view of the canal entrance, the soft tissues below

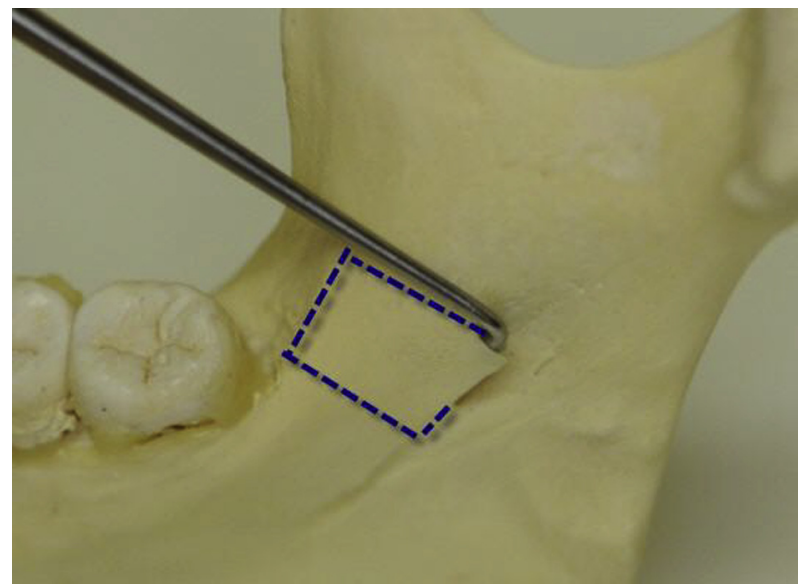

FIGURE 2. The mandibular canal entry is ascertained with a hooklike object. The dashed blue line indicates the part of the bone that will be removed.

Politis et al. Surgical Access to IAN During Cryotherapy. J Oral Maxillofac Surg 2018. 

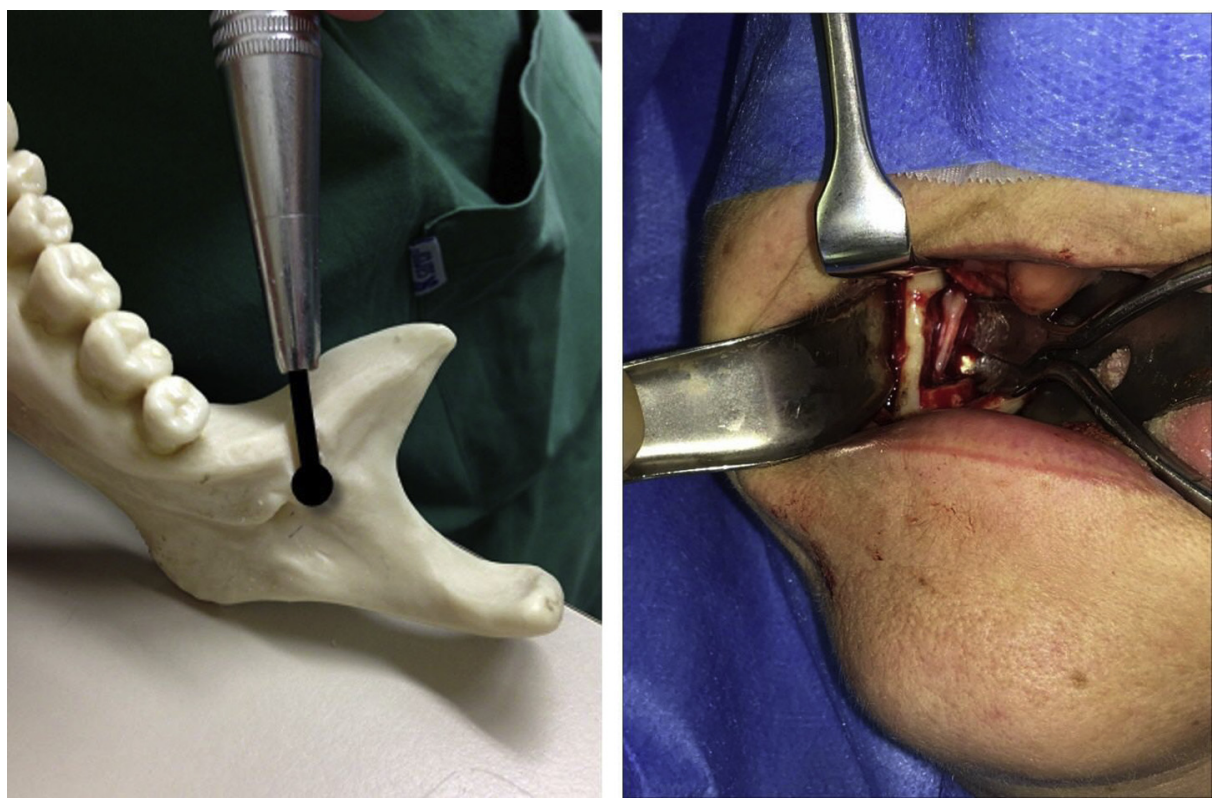

FIGURE 3. Left, Round drills are used to remove the bony camber on the medial side of the ascending ramus, which obstructs a direct view of the mandibular foramen. Right, View inside a patient's mouth in which a Dumbach retractor protects the nerve.

Politis et al. Surgical Access to IAN During Cryotherapy. J Oral Maxillofac Surg 2018.

were mobilized and a second Dumbach retractor was placed below the first retractor.

Next, a cut was made in the cortical bone approximately $1 \mathrm{~cm}$ below the upper surgical bone margin with the piezotome. The cut passed through the cortex, but no deeper than the cortex (Fig 4). (The piezotome was the same one used for the lower- border cut in the bilateral sagittal split osteotomy technique.)

After the bone cuts were performed with the piezotome, the bone fragment was removed with a freer or a molt. This procedure freed the IAN over an extended area; the lower part was liberated from the bony canal and could be readily accessed with a cryoprobe (Fig 5).
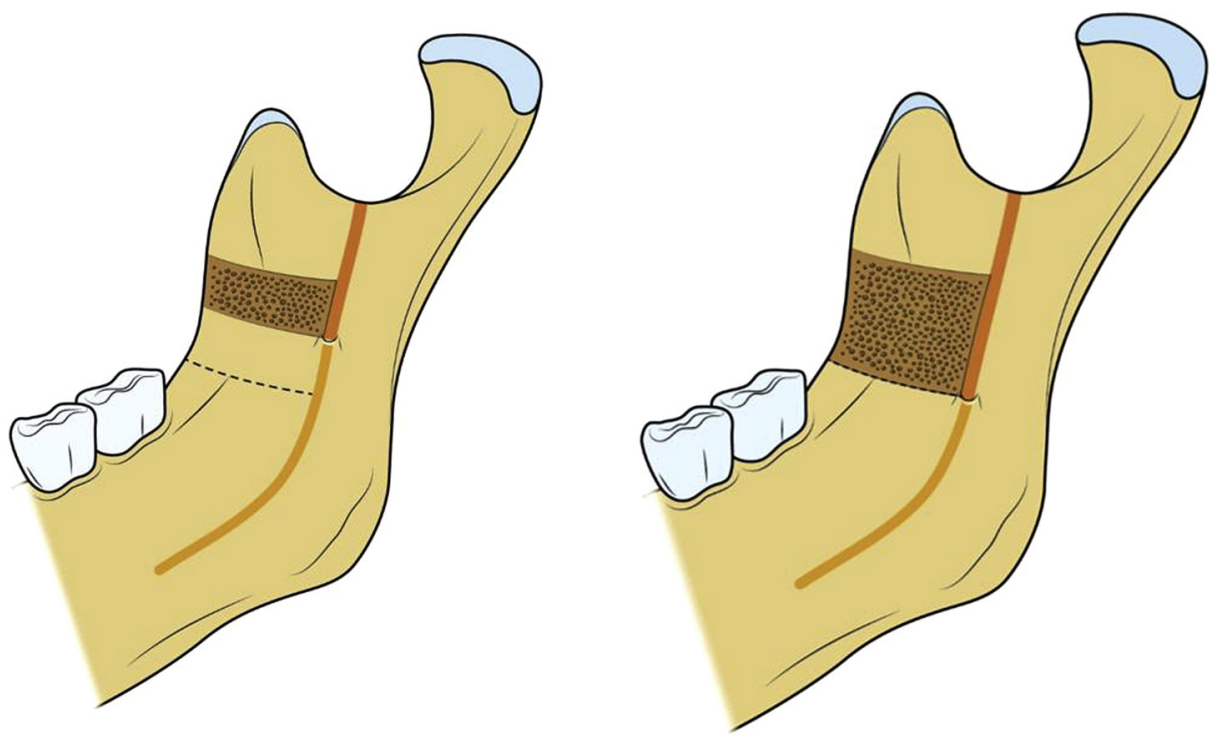

FIGURE 4. Lateral views of the mandible show (leff) locations of the bone cuts relative to the mandibular canal and (right) mobilization of the inferior alveolar nerve.

Politis et al. Surgical Access to IAN During Cryotherapy. J Oral Maxillofac Surg 2018. 


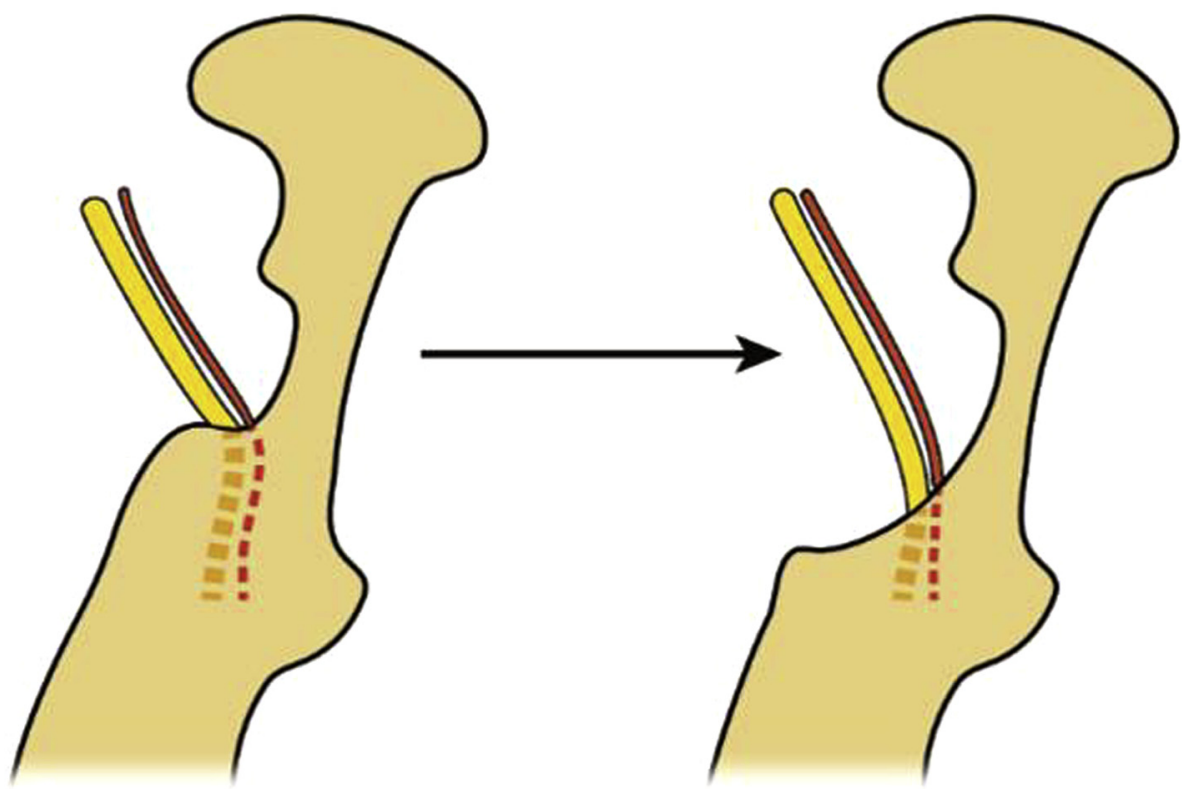

FIGURE 5. The lingula of the mandible and liberation of the inferior alveolar nerve (yellow) and artery (red) over an extended area.

Politis et al. Surgical Access to IAN During Cryotherapy. J Oral Maxillofac Surg 2018.

The Dumbach retractor was repositioned to bring the nerve anterior to the retractor for a direct surgical view (Fig 6).

It is important to keep in mind that the inferior alveolar artery lies directly posterior to the IAN. With the thick cryoprobe, the nerve was frozen for approximately 2 minutes at $-89^{\circ} \mathrm{C}$ (Fig 7 ). This freezing procedure was repeated 3 times.

\section{Discussion}

In 2014, Agbaje et $\mathrm{al}^{11}$ described a surgical technique that ensured safe access to the mandibular nerve at the infratemporal fossa. Although that surgical technique provided sufficient access for orthognathic surgery, wider access was necessary for recurrent cryotherapy procedures at the lingula to avoid jeopardizing the integrity of the nerve. Cryotherapy safeguards the anatomic integrity of the nerve, although freezing causes deep neurapraxia. It produces a reliable, prolonged, and reversible nerve block with no aggravation of symptoms. ${ }^{9}$ A wide surgical access technique prevents the development of postoperative neuropathic pain from damage to the IAN during the surgical procedure. This technique is different from other surgical procedures, such as posterior fossa surgery or partial rhizotomy, that many patients are unwilling or are medically unfit to undergo but are prepared to accept peripheral procedures to give relief. ${ }^{13}$ Most peripheral techniques, such as injection of alcohol, phenol, or lignocaine, neurectomies, and peripheral radiofrequency thermolysis present with associated complications, such as sensory loss, neuritis, or neuroma formation. ${ }^{13}$

The authors described a technique that provided wide intraoral surgical access to enhance procedures

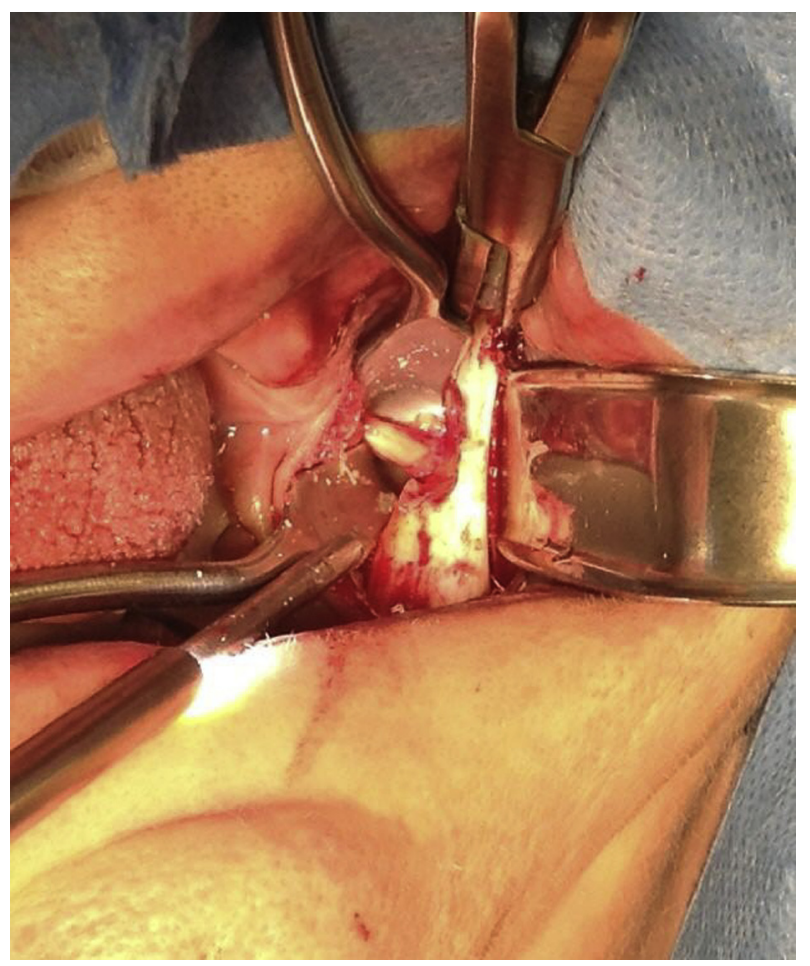

FIGURE 6. The nerve is positioned anterior to the retractor. Politis et al. Surgical Access to IAN During Cryotherapy. J Oral Maxillofac Surg 2018. 

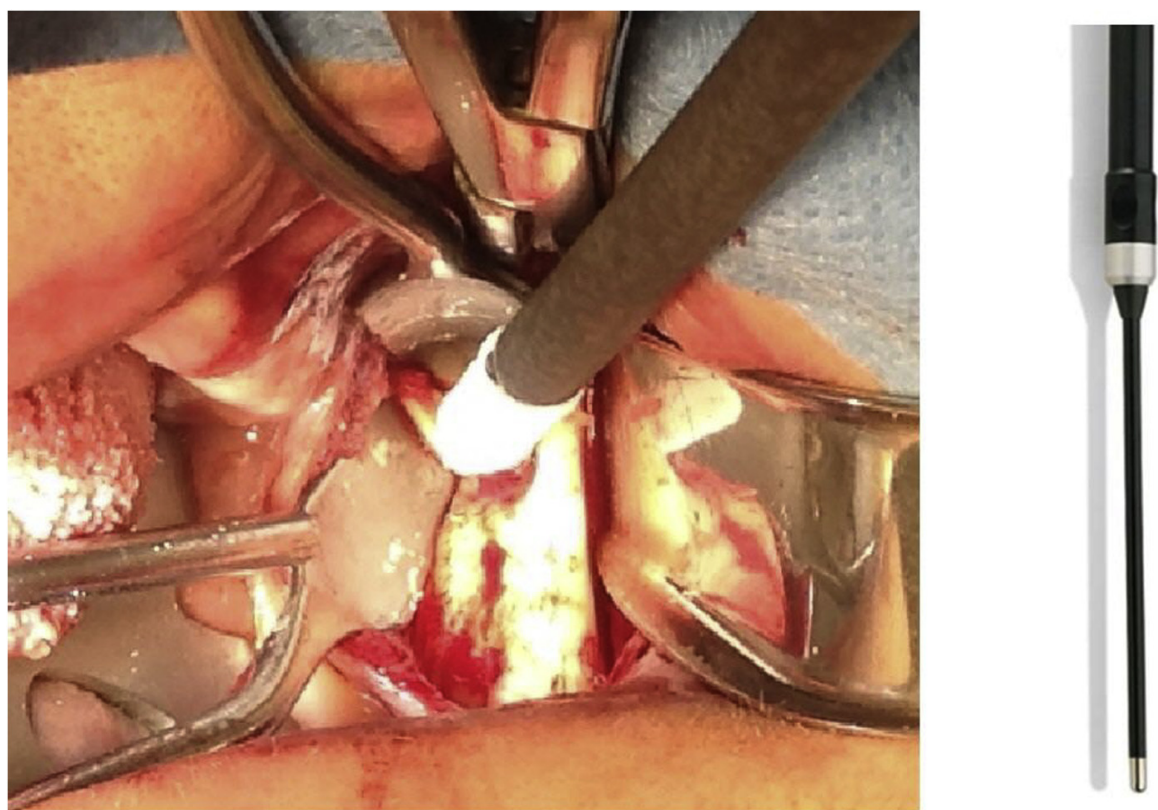

FIGURE 7. Left, The position of the retractor, nerve, and cryoprobe. Right, View of the entire cryoprobe.

Politis et al. Surgical Access to IAN During Cryotherapy. J Oral Maxillofac Surg 2018.

involving the region below the fibrotic region, and this technique facilitated mobilization of the IAN from its bony canal. This technique also facilitated the ability to distinguish the lingual nerve from the IAN. One of the authors (C.P.) has used this technique in cryotherapy procedures. In all cases, visual control of the nerve enabled visual confirmation that no damage was incurred to other structures around the IAN.

\section{References}

1. De Toledo IP, Conti RJ, Fernandes M, et al: Prevalence of trigeminal neuralgia: A systematic review. J Am Dent Assoc 147:570, 2016

2. Maarbjerg S, Gozalov A, Olesen J, Bendtsen L: Trigeminal neuralgia-A prospective systematic study of clinical characteristics in 158 patients. Headache 54:1574, 2014

3. Benoliel R, Zini A, Khan J, et al: Trigeminal neuralgia (part II): Factors affecting early pharmacotherapeutic outcome. Cephalalgia 36:747, 2016

4. Zhang J, Yang M, Zhou M, et al: Non-antiepileptic drugs for trigeminal neuralgia. Cochrane Database Syst Rev 12: CD004029, 2013
5. Goldhahn WE: [Experiences with the Janetta operation in patients with trigeminal neuralgia]. Zentralbl Neurochir 46: 233, 1985 (in German)

6. Li ST, Wang X, Pan Q, et al: Studies on the operative outcomes and mechanisms of microvascular decompression in treating typical and atypical trigeminal neuralgia. Clin J Pain 21:311, 2005

7. Lloyd JW, Barnard JD, Glynn CJ: Cryoanalgesia. A new approach to pain relief. Lancet 2:932, 1976

8. Politis C, Adriaensen H, Bossuyt M, Fossion E: The management of trigeminal neuralgia with cryotherapy. Acta Stomatol Belg 85: 197,1988

9. Poon CY: Cryotherapy in the management of trigeminal neuralgia: A review of the literature and report of three cases. Singapore Dent J 23:49, 2000

10. Barnard D: The use of cryotherapy in the management of paroxysmal trigeminal neuralgia. Oral Surg Oral Med Oral Pathol 59: 463,1985

11. Agbaje JO, Sun Y, Lambrichts I, et al: Safe surgical access to the mandibular nerve at the infratemporal fossae. J Craniofac Surg $25: 1454,2014$

12. Falter B, Schepers S, Vrielinck L, et al: Occurrence of bad splits during sagittal split osteotomy. Oral Surg Oral Med Oral Pathol Oral Radiol Endod 110:430, 2010

13. Zakrzewska JM, Nally FF: The role of cryotherapy (cryoanalgesia) in the management of paroxysmal trigeminal neuralgia: A six year experience. Br J Oral Maxillofac Surg 26:18, 1988 